\title{
Réflexion sur la recherche biomédicale
}

\author{
Henri-Géry Hers
}

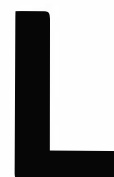

a recherche médicale, au même titre que toute autre recherche scientifique, se divise en fondamentale et appliquée. La première s'efforce de comprendre comment l'homme normal fonctionne et comment on peut expliquer les maladies sur la base d'un dérèglement du processus normal. La seconde relève de la technologie et son souci primordial est de guérir la maladie ou, tout au moins, de soulager le malade, ce qui peut certes se faire sans nécessairement comprendre les mécanismes en jeu ; c'est bien là l'aspect le plus directement utile à la société et qui trouve toute sa justification en luimême. L'histoire nous montre en effet que, bien souvent, les grands progrès de la thérapeutique ont précédé, parfois de plus d'un siècle, la compréhension de leur mécanisme. C'est ainsi que Jenner avait découvert la vaccination antivariolique bien longtemps avant qu'on ne sache ce qu'était un anticorps et que les chimistes de la firme Bayer avaient découvert l'action anti-inflammatoire de l'aspirine longtemps avant que Vane ne décrive sa propriété d'inhiber la cyclo-oxygénase et d'empêcher ainsi la synthèse des prostaglandines.

Pour faire comprendre les rôles respectifs de la recherche appliquée et de la recherche fondamentale, il est particulièrement approprié de considérer le cas bien connu de la théra- peutique anticancéreuse, domaine où il semble que la société soit prête à investir des fonds illimités pour arriver à une solution du problème. La recherche anticancéreuse procède en effet de plusieurs stratégies. La première est fondée sur la technologie et consiste à appliquer au traitement du cancer tout ce que nous connaissons en biologie, en chimie et en physique. Cette approche a permis de mettre au point différents traitements radiothérapiques et chimiothérapiques qui ont notablement réduit la mortalité d'un certain nombre de cancers. Il est incontestable, cependant, que ce succès n'est que partiel et que l'on est encore loin du but, simplement parce que nous comprenons encore mal ce qu'est le cancer et que ce que nous en connaissons ne nous permet pas d'en guérir toutes les formes. Pour parvenir à ce but, la seule solution est d'affronter l'inconnu, c'est-à-dire d'amplifier l'ensemble de nos connaissances de base, tout en restant conscients du fait que nous ignorons complètement de quel domaine de la science sortira la solution, si solution il y a, et que, de plus, il n'y a pas de limites de temps ni d'argent qui puissent être définies pour l'aboutissement de ce type de recherche.

Cette différence entre les potentialités respectives de la recherche appliquée et de la recherche fondamentale est particulièrement bien illustrée par le succès très différent remporté par deux présidents des États-Unis d'Amérique qui, l'un et l'autre, avaient lancé un défi à la science de leur pays. Le président Kennedy, outré par le lancement du Spoutnik soviétique, avait promis d'envoyer un Américain sur la Lune dans les dix années à venir et nous savons tous que l'astronaute Armstrong a effectivement "aluni " le 20 juillet 1969. Kennedy a pu tenir ce défi parce que tout ce qu'il fallait connaître pour aller sur la Lune était connu et que, pour réaliser le projet, il suffisait d'y consacrer le temps et l'argent nécessaires. Un peu plus tard, Nixon, voulant imiter Kennedy, s'était lui aussi donné dix ans pour arriver à la guérison du cancer, mais il fut bien incapable de tenir son défi, car, contrairement à ce que nous venons de dire de la conquête de l'espace, les connaissances de base nécessaires à la guérison du cancer étaient tout à fait insuffisantes. De toute évidence, Nixon ignorait la différence entre l'application des données connues et la découverte de l'inconnu. L'argent qu'il a donné a bien heureusement mené à diverses découvertes, mais qui n'avaient pas nécessairement de relation avec le cancer. Ce qui est vrai du cancer l'est de beaucoup d'autres maladies actuellement incurables, comme les affections congénitales, la démence sénile, le SIDA et bien d'autres. C'est également vrai 
de problèmes non médicaux, tels que celui des nouvelles sources d'énergie, de la prévision du temps, de la pollution, etc.

Une autre façon de forcer l'inconnu, souvent utilisée par l'industrie pharmaceutique, est la méthode empirique d'une chasse à l'aveugle. Elle consiste à tester l'action biologique, notamment antitumorale, d'innombrables substances prises au hasard, puisque c'est bien le hasard qui fait apparaître ce dont nous ne pouvons soupçonner l'existence. Cette méthode a parfois donné des résultats remarquables, bien que pas toujours conformes au but poursuivi. Elle a, par exemple, permis la découverte de l'allopurinol, un analogue des bases puriques, dont on attendait qu'il inhibe la multiplication cellulaire et qui s'est révélé très actif contre la goutte, mais dépourvu de toute action antitumorale.

Il n'est donc pas toujours facile d'établir la frontière entre recherche fondamentale et recherche appliquée et c'est de cette heureuse incertitude que bénéficient les scientifiques fondamentalistes à une époque où l'on ne souhaite soutenir que la technologie. Les exemples abondent en effet de progrès technologiques dus à des fondamentalistes et inversement, et c'est à juste titre que d'aucuns prétendent que la distinction entre sciences de base et sciences appliquées est fallacieuse. En fait, chacun des deux groupes se caractérise essentiellement par sa motivation. En recherche appliquée, seul compte le but à atteindre et, dans les plus mauvais cas, le chercheur n'a pas la permission de s'égarer au fil des observations fortuites qui lui auraient permis de pénétrer l'inconnu, puisque celuici, par définition même, ne pouvait être inclus dans le programme. Une recherche aussi strictement orientée exclut toute véritable découverte. En science fondamentale au contraire, le projet est l'occasion de développer des recherches dont les plus beaux jours sont ceux où l'on trouve ce que l'on ne cherchait pas. De tels progrès sont les seuls qui déplacent la frontière entre le connu et l'inconnu et ment inoubliable d'être un " découvreur".

Si la motivation est effectivement ce qui distingue la recherche fondamentale de la recherche appliquée, elle est souvent, et bien remarquablement, de peu d'influence sur le résultat acquis. Dans mon cas personnel, j'eus l'immense privilège de débuter ma carrière de chercheur sous la direction de Christian de Duve et je ne pourrais trop souligner en passant que ce que l'on apprend d'un Maître, ce n'est pas tant la science ellemême, car on la trouve dans les livres, que la rigueur avec laquelle il faut la considérer et l'esprit critique qui permet de percevoir, dans une information devenue trop abondante, ce qu'il y a lieu de croire et ce dont il y a lieu de douter. C'est aussi de son Maître que le chercheur hérite son sujet de recherche et le domaine de la science dans lequel il restera le plus souvent confiné durant toute sa carrière. Christian de Duve voulait trouver le mode d'action de l'insuline, sujet grandiose s'il en fut et qui a hanté l'esprit de milliers de chercheurs de par le monde, sans avoir jusqu'à présent été résolu. De Duve pensait que l'insuline favorisait la synthèse du glycogène hépatique au niveau de l'interconversion entre le glucose et le glucose-6-phosphate. C'est la raison pour laquelle nous entreprîmes l'étude de la glucose-6-phosphatase. Ceci ne nous apprit rien sur l'insuline mais nous permit de caractériser l'enzyme et surtout de montrer sa liaison au réticulum endothélial, fait que personne n'aurait pu soupçonner et dont personne, encore aujourd'hui, ne comprend la raison d'être. C'est pourtant ce petit fait qui conduisit de Duve à une étude approfondie de la localisation subcellulaire des enzymes et finalement à la découverte des lysosomes. Pour ma part, la connaissance que j'avais acquise de la glucose-6-phosphatase fut l'occasion de m'intéresser aux glycogénoses et de reprendre ainsi contact avec la médecine clinique.

C'est encore dans l'espoir de découvrir le mode d'action de l'insuline que j'ai étudié, sans succès, la glucokinase et, secondairement, à titre de contrôle, la fructokinase et que, de là, je suis arrivé au métabolisme du fructose. Celui-ci non plus n'avait rien à voir avec le mode d'action de l'insuline et ce n'est que très indirectement que j'ai pu faire une petite contribution, sinon à l'insuline tout au moins au diabète, en décrivant l'aldose réductase. Je découvris cette enzyme, alors que je ne pensais plus à l'insuline, en recherchant comment le glucose donne naissance au fructose présent dans le plasma séminal. C'est en effet l'aldose réductase et l'excès de sorbitol formé par cette enzyme chez le diabétique que l'on incrimine dans la pathogénie de certaines des complications du diabète et il n'est pas exclu que, dans un proche avenir, un inhibiteur de l'aldose réductase ne rende de grands services aux malades atteints de diabète. Ce fut donc là ma principale contribution à l'insuline. Je la fis sans en être conscient et ne participai en rien à son développement.

Tout ceci pour vous dire que le projet de recherche, et notamment celui que l'on écrit pour demander un soutien financier, est sans importance quant au résultat acquis. Sur quelle base faut-il dès lors distribuer l'argent disponible pour la recherche. Il faut bien sûr ne pas procéder dans le secret, mais que cette disponibilité des fonds soit largement diffusée et que des comités compétents fassent la sélection des bénéficiaires. Il y a lieu toutefois de se demander comment ces comités doivent procéder. Les demandeurs leur soumettent en général d'abondants documents qui comprennent la description du projet, lequel porte sur plusieurs années, le curriculum vitae des demandeurs et finalement la liste de ce qui est demandé. Après ce que je viens de vous raconter, vous ne serez pas très surpris si je vous dis qu'à mes yeux le projet lui-même n'a que très peu d'importance. Un projet, en effet, se laisse écrire. Les auteurs y décrivent, certains avec beaucoup de talent, ce qu'ils espèrent trouver et j'ai toujours été surpris tant par la candeur des auteurs que par la naïveté de ceux qui les lisent.

Vous ne serez donc pas trop scandalisés lorsque je vous aurai avoué que, 
dans les nombreuses commissions de sélection dont j'ai fait partie, je n'ai jamais lu que très superficiellement les programmes qui m'étaient soumis. En revanche, j'ai toujours considéré avec la plus grande attention la production scientifique antérieure des demandeurs, et j'ai donné un avis favorable à ceux qui pouvaient montrer qu'ils avaient l'habitude d'être productifs. En procédant de la sorte, je n'ai fait qu'appliquer la règle qui dit : "En recherche fondamentale, ce sont les hommes que l'on soutient, tandis qu'en recherche appliquée, ce sont les projets." Encore ici, la deuxième partie de cette règle ne s'applique-t-elle qu'à la recherche appliquée dont les limites sont très strictement définies et où il n'y a pas de surprise à attendre. La règle ne s'applique plus dès qu'un projet, présenté comme technologique par ses auteurs à cause des retombées pratiques potentielles, comporte un certain degré d'incertitude, car nul ne peut échapper à cette évidence que l'inconnu est improgrammable. A nouveau, de tels projets doivent être jugés en fonction des hommes qui les proposent bien plus qu'en fonction de leur contenu propre.

Après tout le mal que je vous ai dit des projets, vous pourriez croire que les chercheurs arrivent chaque matin au laboratoire sans savoir ce qu'ils vont y faire, sans programme précis. C'est, bien sûr, le contraire qui se passe. Tout chercheur sérieux a un programme, qui est fait d'expériences susceptibles de donner le plus rapidement possible une réponse non ambiguë à une question correctement posée. Ce programme, qui est le seul authentique, n'est cependant pas vendable à des divers organismes nationaux ou internationaux qu'en terme gaullien j'appellerai "les grands machins ", tels CEE, OMS, Eureka, Esprit et bien d'autres. Et ceci pour plusieurs raisons. La première est que, si le programme a quelque valeur, il doit être entrepris immédiatement et ne peut attendre les nombreux mois, voire les années, que prendront toutes les formalités requises pour obtenir un support officiel. La deuxième èst que ces programmes sont éminemment flexibles $m / s n^{\circ} 2$, vol. 7 , février 91 et constamment remis en question, et qu'ils ne portent guère de façon précise que sur une période de quelques mois ou au maximum une année. La troisième, qui vous surprendra peutêtre, est une question de pudeur, car il est certaines hypothèses audacieuses que des chercheurs gardent au fond de leur cœur sans les révéler, soupçonnant que personne ne les croira, avant qu'ils n'aient pu en apporter la preuve définitive. C'est ainsi que Jenner garda secrète pendant plusieurs années son hypothèse de vaccination contre la variole, craignant que ses collègues ne se moquent de lui. L'idée d'inoculer une maladie du bétail à l'homme pouvait en effet paraître quelque peu incongrue.

Ce qui fait la qualité du programme individuel du chercheur, c'est la pertinence et l'originalité de la question posée et surtout la profondeur jusqu'à laquelle l'expérimentateur est décidé à s'avancer pour résoudre son problème. Pour prendre un exemple qui m'est familier, vous savez que les lysosomes, qui furent découverts il y a un peu plus de trente ans par Christian de Duve presque sous mes yeux, se trouvent en fait dans à peu près toutes les cellules et auraient donc pu être découverts par n'importe quel biologiste, pour peu qu'il ait osé quitter les lieux sécurisants du déjà-connu. Pour trouver du neuf, il faut en effet oser le chercher et ce n'est pas là une question de chance, laquelle a très peu d'importance, mais bien d'habitude dans la façon de considérer les problèmes.

Le temps est maintenant venu de conclure en vous disant que beaucoup de ceux qui veulent du bien à la recherche la maltraitent parce qu'ils la connaissent mal. J'ai voulu vous montrer, de l'intérieur, comment la recherche fonctionne et souligner surtout la vanité des grands programmes et la valeur des hommes. Je crois en effet que l'on obtiendrait un rendement maximal de l'argent consacré à la recherche si l'on se contentait de le distribuer, selon leurs besoins, à tous ceux qui se sont montrés productifs, et cela sans leur demander de programme. Un système aussi simple mettrait probablement au chômage un grand nombre d'administratifs. C'est la raison pour laquelle je crains bien qu'il ne soit jamais accepté

Extrait de l'allocution prononcée le 7 décembre 1990 à l'Université Claude-Bernard à Lyon à l'occasion de la remise du titre de docteur honoris causa.

\section{H.G. Hers}

Professeur émérite à l'université catholique de Louvain. Laboratoire de chimie physiologique, Université catholique de Louvain et International Institute of Molecular and Cellular Pathology, 75, avenue Hippocrate, B 1200 Bruxelles, Belgique.

\section{TIRÉS A PART}

H.G. Hers.

\section{PRIX LOUIS JEANTET}

Le Professeur Pierre Chambon, de Strasbourg, est le lauréat 1991 du Prix Louis Jeantet, associé à Frank Grosveld (Londres, GB) et à Hug Pelham (Cambridge, GB). Ce sont trois illustres généticiens moléculaires qui se voient distingués par ce prix de 1,95 millions de francs suisses, créé grâce à la fondation d'un riche industriel français dont toute l'activité s'est déroulée à Genève.

Le but du Prix Louis Jeantet est de récompenser les meilleurs scientifiques d'Europe Occidentale. Dans le cas présent, les trois lauréats ont apporté une contribution essentielle à l'élucidation du fonctionnement des gènes eucaryotes.

Pierre Chambon a participé (et participe) à la majorité des progrès des connaissances en ce domaine, depuis les ARN polymérases jusqu'au contrôle des gènes par les hormones stéroïdes, en passant par la découverte des introns, de leur structure, des enhancers, etc. 